\title{
Entangled Andreev pairs and collective excitations in nanoscale superconductors
}

\author{
A. LEVY YEYATI ${ }^{* *}$, F. S. BERGERET ${ }^{1}$, A. MARTÍN-RODERO1 AND T. M. KLAPWIJK² \\ ${ }^{1}$ Departamento de Física Teórica de la Materia Condensada C-V, Universidad Autónoma de Madrid, E-28049 Madrid, Spain \\ ${ }^{2}$ Kavli Institute of Nanoscience, Delft University of Technology, Lorentzweg 1, 2628 CJ Delft, The Netherlands \\ *e-mail: a.l.yeyati@uam.es
}

Nanoscale superconductors connected to normal metallic electrodes provide a potential source of entangled electron pairs $^{1-5}$. Such states would arise from the splitting of Cooper pairs in the superconductor into two electrons with opposite spins, which then tunnel into different leads by means of a process known as crossed Andreev reflection (refs 6-8). In an actual system, the detection of these processes is hindered by the elastic transmission of individual electrons between the leads, which yields an opposite contribution to the non-local conductance. Here we demonstrate that low-energy collective excitations, which appear in superconducting structures of reduced dimensionality ${ }^{9}$, can have a significant influence on the transport properties of this type of hybrid nanostructure. When an electron tunnels into the superconductor it can excite such low-energy excitations that alter the balance between the different electronic processes, leading to a dominance of one over the other depending on the spatial symmetry of these excitations. These findings help to clarify some intriguing experimental results and provide future strategies for the detection of entangled electron pairs in solid-state devices for quantum computation.

A generic set-up for the study of non-local transport through a superconductor is shown in Fig. 1a. It represents a superconducting region attached to three normal electrodes. Two of the leads (labelled 1 and 2 in Fig. 1a) are used to inject a current while the voltage drop is measured on the third one. The two basic microscopic processes contributing to the non-local conductance are illustrated in Fig. 1c,d. In the case of elastic cotunnelling (EC) processes, the injected electron tunnels elastically into the third lead, whereas in the case of crossed Andreev reflection (CAR) processes, it combines with an electron emerging from the third lead to form a Cooper pair in the superconductors. The probability of these processes decays exponentially on the scale of the superconducting coherence length, $\xi$, which can range between 10 and $100 \mathrm{~nm}$ for typical superconductors used in experiments ${ }^{10,11}$. On the other hand, the two processes yield opposite contributions to the non-local conductance (conventionally the CAR contribution is taken as positive) and, as demonstrated by previous theoretical studies ${ }^{12-14}$, tend to cancel each other in the case of Bardeen-Cooper-Schrieffer (BCS) superconductors weakly coupled to non-magnetic leads. Surprisingly, recent experiments by Russo et al. ${ }^{11}$ have shown that even in this case the subgap non-local conductance can be appreciably large, exhibiting an intriguing behaviour in which either process can dominate depending on the energy of the injected electrons. This behaviour cannot be accounted for by the existing non-interacting theories.

The importance of interactions in breaking the balance between EC and CAR processes can be understood by considering the case where the superconducting region is sufficiently small and can be characterized by a finite charging energy, $E_{\mathrm{c}}=e^{2} /(2 C)$, where $C$ denotes the corresponding capacitance. As shown in Fig. 1c, EC processes take place through a virtual state that will be shifted upwards by the Coulomb energy. The process, however, would not be blocked for any value of the applied voltage, as the initial and final states have the same energy. In contrast, CAR processes demand that two electrons tunnel into the superconducting region forming a Cooper pair, a process which requires an extra energy of $4 E_{\mathrm{c}}$. Thus, the non-local conductance has a finite (negative) value for a voltage, $V$, smaller than $4 E_{\mathrm{c}} / e$ where EC processes dominate, whereas it vanishes for $e V>4 E_{c}$ when both processes tend to cancel each other. These predictions coincide with the results of a more detailed calculation on the basis of the theory discussed below. They also provide a first simple example in which the role of interactions could be tested experimentally.

For a quantitative analysis of the influence of interactions we describe the system by a hamiltonian, $\hat{H}=\hat{H}_{\mathrm{S}}+\hat{H}_{\text {leads }}+\hat{H}_{T}+\hat{H}_{\text {env }}$. The first three terms correspond to the electronic degrees of freedom. $\hat{H}_{\mathrm{S}}$ is the usual BCS hamiltonian for the superconducting region and $\hat{H}_{\text {leads }}$ describes the normal leads that we label with an index $n$. The tunnelling of electrons between the leads and the superconductor is described by $\hat{H}_{T}=\sum_{n} \hat{H}_{T, n}$, with

$$
\hat{H}_{T, n}=\sum_{\sigma} \int_{S_{n}} \mathrm{~d}^{2} r\left[v_{n} \hat{\psi}_{l n, \sigma}^{\dagger}(\mathbf{r}) \hat{\psi}_{s n, \sigma}(\mathbf{r}) \mathrm{e}^{i \hat{\phi}_{n}(\mathbf{r})}+\text { h.c. }\right],
$$

where the integral is taken over the junction area $S_{n}, \hat{\psi}_{l n, \sigma}^{\dagger}(\mathbf{r})$ and $\hat{\psi}_{s n, \sigma}^{\dagger}(\mathbf{r})$ are electron creation operators on the two sides of the junction and $\hat{\phi}_{n}(\mathbf{r})$ is the corresponding phase drop that is conjugate to the charge density on the junction $\hat{Q}_{n}(\mathbf{r})$, that is, $\left[\hat{\phi}_{n}(\mathbf{r}), Q_{n}\left(\mathbf{r}^{\prime}\right)\right]=i e \delta\left(\mathbf{r}-\mathbf{r}^{\prime}\right)$. h.c. is the hermitian conjugate. The dynamics of these phase operators is determined by the hamiltonian $\hat{H}_{\text {env }}$ describing the electromagnetic environment that characterizes the actual experimental set-up. It is important to note that, owing to the typical distances between the leads in the experiments, which cannot be much larger than $\xi$, correlations 

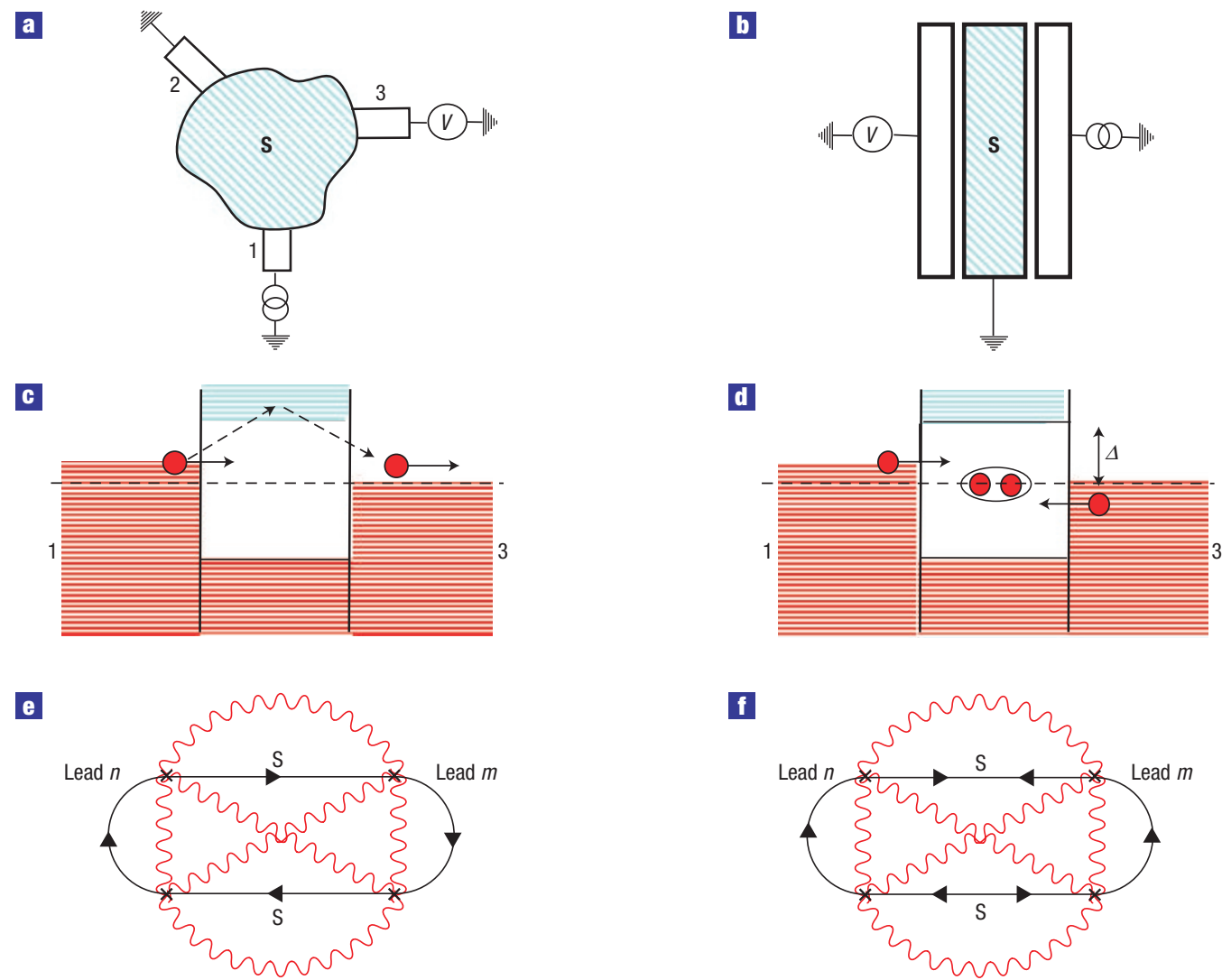

Figure 1 Typical set-ups and basic microscopic processes in non-local transport through a superconductor. a, Schematic representation of a generic multiterminal geometry where a superconducting region (S) is coupled to several metallic leads $(1,2,3)$. b. Double planar normal/superconducting/normal junction geometry studied in ref. 11. c,d, Pictorial description of EC (c) and CAR (d) processes in energy space. e,f, Feynman diagrams corresponding to the calculation of the non-local conductance to fourth order taking into account interactions mediated by the electromagnetic environment. Crosses indicate the tunnelling events, solid lines with an arrow represent the normal and anomalous propagators and wavy lines indicate phase correlators.

between voltage fluctuations on different junctions cannot be neglected, that is, correlation functions of the type $\left\langle\hat{\phi}_{n} \hat{\phi}_{m}\right\rangle$, with $n \neq m$ are non-zero. In addition, the reduced dimensions of the superconducting region can give rise to the presence of collective modes within the superconducting gap, which can dominate the behaviour of the phase correlations.

To obtain the transport properties of this model we use the Keldysh-Nambu Green functions formalism, which is well adapted to analyse non-equilibrium situations in the presence of superconductivity (for details see Supplementary Information). The contributions from EC and CAR processes to the non-local conductance $G_{n m}$, that is, the variation of the current through lead $n$ due to a voltage applied on lead $m$, in the tunnel limit is represented by the type of diagrams shown in Fig. 1e,f. The solid lines with an arrow represent the electron propagators, whereas the wavy lines describe the coupling with the environment, that is, they denote the phase correlators of the type $\left\langle\mathrm{e}^{i \hat{\phi}_{n}} \mathrm{e}^{-i \hat{\phi}_{m}}\right\rangle$. Let us first consider the simplest case where the environment can be characterized by a single electromagnetic mode of frequency $\omega_{0}$. We can further assume that the leads are coupled to the superconducting region through point contacts as illustrated in Fig. 2a,b. Two opposite situations can be distinguished depending on the spatial symmetry of the electromagnetic mode under consideration: it can lead to either symmetric or antisymmetric voltage fluctuations on the two junctions. In the symmetric case, for clean BCS superconductors and assuming that $\hbar \omega_{0}$ is much smaller than the superconducting gap, $\Delta$, but larger than the charging charging energy, $E_{c}$, of the tunnel junctions, at zero temperature we obtain (see the Supplementary Information)

$$
\begin{aligned}
G_{n m} \simeq & -\frac{G_{n} G_{m}}{G_{0}} \frac{\mathrm{e}^{-2 R / \xi}}{\left(k_{\mathrm{F}} R\right)^{2}}\left[\cos ^{2}\left(k_{\mathrm{F}} R\right)\right. \\
& \left.-\left(1-z_{0} \theta\left(\hbar \omega_{0}-e V\right)\right) \sin ^{2}\left(k_{\mathrm{F}} R\right)\right] .
\end{aligned}
$$

Here $G_{n(m)}$ is the normal conductance of the junction $n(m), R$ is the distance separating the leads, $k_{\mathrm{F}}$ is the Fermi wavevector, $G_{0}=2 e^{2} / h$ is the conductance quantum and $V$ is the voltage applied on lead $m$. The term proportional to $\cos ^{2}\left(k_{\mathrm{F}} R\right)$ corresponds to the EC contribution, whereas the $\sin ^{2}\left(k_{\mathrm{F}} R\right)$ term arises from CAR processes. The parameter $z_{0}=E_{c} / \hbar \omega_{0}$ measures the coupling to the electromagnetic mode. Expression (1) is the lowest order in $z_{0}$ of the general result for arbitrary values of $z_{0}$ presented in the Supplementary Information. It is worth noting that this expression reproduces the non-interacting result ${ }^{12}$ for $z_{0}=0$, where a complete cancellation between CAR and EC contributions takes place on averaging over the Fermi wavelength scale. For finite but small $z_{0}$, the balance between EC and CAR is broken: for $\mathrm{eV}$ smaller than $\hbar \omega_{0}$ the CAR processes become suppressed and non-local transport is dominated by the EC contribution, whereas for $\mathrm{eV}>\hbar \omega_{0}$ both contributions tend to cancel, as in the non-interacting case. The suppression of the CAR 


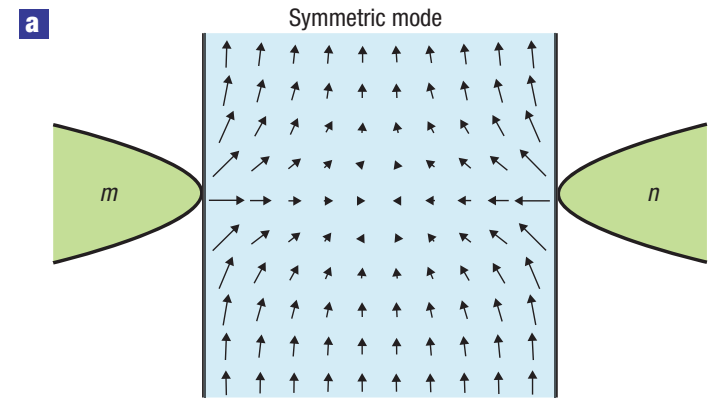

G

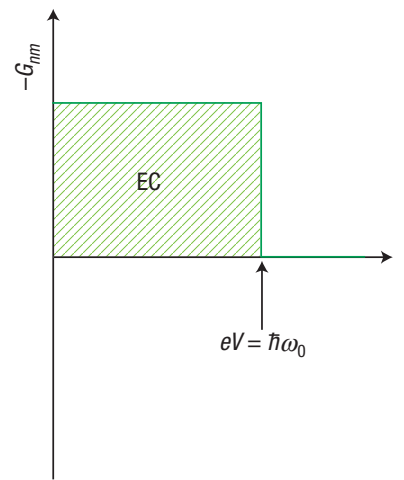

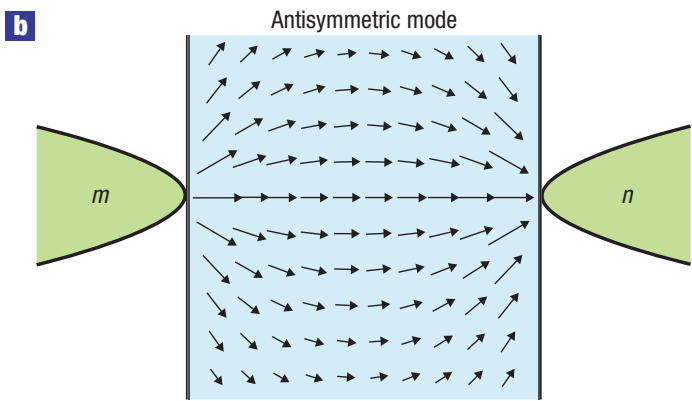

d

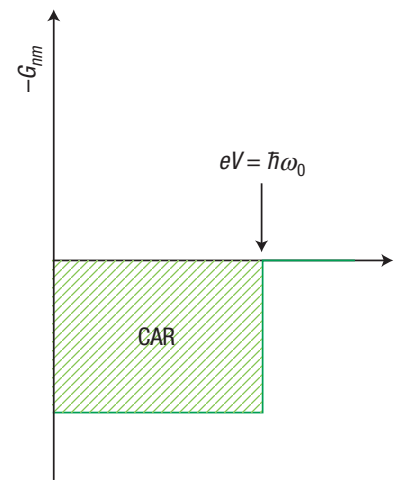

Figure 2 Effect of interactions in the non-local conductance. a,b, Pictorial representation of the effect of interactions mediated by electromagnetic modes of different symmetry on the non-local conductance between two point contacts. The arrows represent the phase gradient within the superconductor. c,d, Whereas symmetric modes tend to suppress CAR processes (c), the antisymmetric ones suppress the EC contribution (d).

contribution is due to the impossibility of such processes to occur without producing a real excitation of the environment, as in the constant charging energy example.

The situation is the opposite in the case of an antisymmetric mode. The analogue of expression to equation (1) for this case is (see the Supplementary Information)

$$
\begin{aligned}
G_{n m} \simeq & -\frac{G_{n} G_{m}}{G_{0}} \frac{\mathrm{e}^{-2 R / \xi}}{\left(k_{\mathrm{F}} R\right)^{2}}\left[\left(1-z_{0} \theta\left(\hbar \omega_{0}-e V\right)\right)\right. \\
& \left.\times \cos ^{2}\left(k_{\mathrm{F}} R\right)-\sin ^{2}\left(k_{\mathrm{F}} R\right)\right],
\end{aligned}
$$

which corresponds to a suppression of EC (instead of CAR) at low voltages. The different effect of symmetric and antisymmetric modes is schematically illustrated in Fig. 2c,d.

The electromagnetic environment in a general experimental situation can be described as a collection of modes. For instance, let us consider the case of a planar geometry similar to the one in the experiments of ref. 11 (represented by Fig. 1b), consisting of a superconducting layer of thickness $d \gtrsim \xi$ coupled to two normal leads by tunnel junctions. For simplicity, we describe them as infinite planes. This situation is characterized by the presence of propagating modes along the superconducting/normal junctions, which can be derived from the following model hamiltonian

$$
\hat{H}_{\mathrm{env}}=\left(\frac{\hbar}{2 e}\right)^{2} \int \mathrm{d}^{3} r \frac{(\nabla \hat{\phi})^{2}}{2 L d}+\int \mathrm{d}^{2} r \frac{1}{2 C_{\square}}\left(\hat{Q}_{L}^{2}+\hat{Q}_{R}^{2}\right),
$$

where the term containing the phase gradient describes the kinetic energy associated with the supercurrents in the superconducting film, $L$ being its total inductance, whereas the second term is the Coulomb energy of the charge accumulated on the junctions, denoted by $\hat{Q}_{L, R}$. The junctions are assumed to be symmetric with capacitance $C_{\square}$ per unit area and with cross-section $\&$. In writing this hamiltonian, we are also assuming that longrange Coulomb interactions are screened by the normal electrodes acting as ground planes ${ }^{15}$. The low-energy modes that result from this model correspond to symmetric and antisymmetric voltage fluctuations on the junctions, with dispersion relations $\omega_{1}(\mathbf{q})=c_{s} \sqrt{(q \tanh q d) / d}$ and $\omega_{2}(\mathbf{q})=c_{s} \sqrt{q /(d \tanh q d)}$, where $\mathbf{q}$ is the wavevector in the direction parallel to the film and $c_{s}=1 / \sqrt{L C_{\square}}$. Note that for small $q$ the symmetric mode exhibits a linear dispersion with phase velocity $c_{s}$, whereas the antisymmetric one tends to a finite frequency $\omega_{0}=c_{s} / d$ in the limit $q \rightarrow 0$. This description of the low-energy modes captures the essential features of a detailed calculation based on Maxwell equations for the double planar junction geometry (see the Supplementary information).

We can roughly estimate the order of magnitude of the parameters in $\hat{H}_{\text {env }}$ for the experimental situation. Thus, $C_{\square}$ can be obtained from the typical charging energy for an oxide barrier tunnel junction $E_{\mathrm{c}} \& \sim 1 \mu \mathrm{eV} \times \mu \mathrm{m}^{2}$ and $L$ can be estimated as $\mu_{0} \lambda^{2} / d$, where $\lambda$ is the field penetration depth ${ }^{16}$. The actual value of $\lambda$ for a $\mathrm{Nb}$ film is strongly dependent on its thickness, degree of disorder and it is also influenced by the properties of the nonsuperconducting substrate on which it is deposited ${ }^{17}$. Reported values range between $100 \mathrm{~nm}$ and $1 \mu \mathrm{m}$ for $d \sim 10-100 \mathrm{~nm}$ (refs 17,18). Within this range of parameters, the lowest energy of the antisymmetric mode $\hbar \omega_{0}$ can be of the order of the superconducting gap in $\mathrm{Nb}$, even for the smaller film thickness analysed in ref. 11 . 

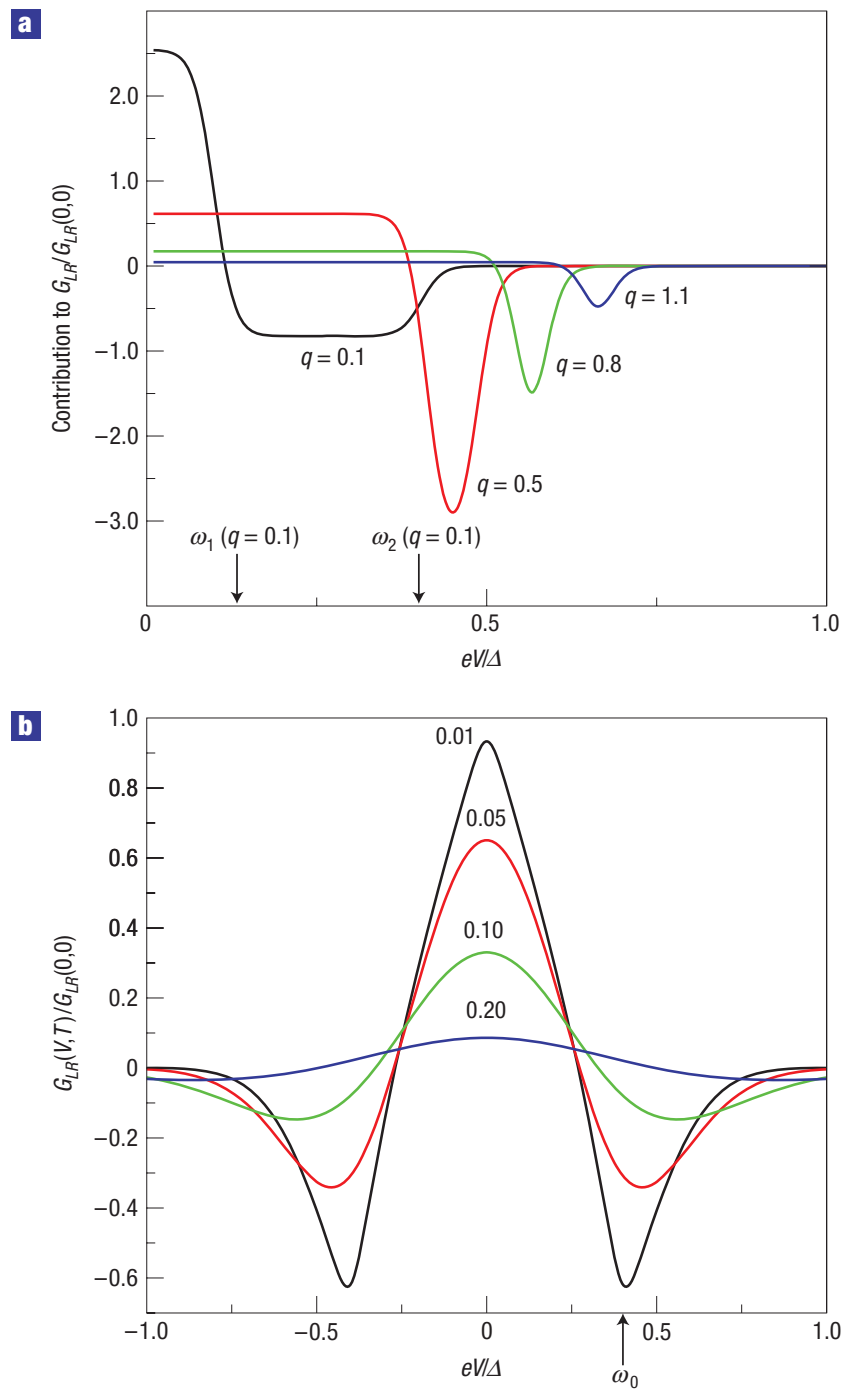

Figure 3 Non-local conductance in double planar junction geometry. a, Contribution to the non-local conductance from modes with a given wavevector in the double planar junction geometry for $k_{\mathrm{B}} T=0.01 \Delta$ and $\hbar \omega_{0}=0.4 \Delta$. The values of $q$ are given in units of $\Delta / \hbar c_{s}$. The arrows indicate the energy of symmetric and antisymmetric modes for $q=0.1$. $\mathbf{b}$, Temperature and voltage dependence of the total non-local conductance. The temperature values are given in units of $\Delta / k_{\mathrm{B}}$. The arrow indicates the energy $\hbar \omega_{0}$ for the lowest antisymmetric mode.

To obtain the non-local conductance, $G_{L R}$, measured at the left interface when a voltage, $V$, is applied on the right junction, we extend the theory developed for the single mode case, linearizing with respect to the coupling parameters $z_{1,2}(\mathbf{q})=E_{\mathrm{c}} / \hbar \omega_{1,2}(\mathbf{q})$, which is justified for the range of parameters estimated above. We thus obtain

$$
\begin{aligned}
G_{L R}= & 4 \pi \frac{G_{L} G_{R}}{\delta k_{\mathrm{F}}^{2} G_{0}} \mathrm{E} 1(2 d / \xi) \sum_{\mathbf{q}, \alpha=1,2}(-1)^{\alpha} z_{\alpha}(\mathbf{q})\left[\left(N\left(\omega_{\alpha}(\mathbf{q})\right)+1\right)\right. \\
& \left.\times\left(F\left(\omega_{\alpha}(\mathbf{q})\right)+1\right)+N\left(\omega_{\alpha}(q)\right)\left(F\left(-\omega_{\alpha}(q)\right)+1\right)\right],
\end{aligned}
$$

where $G_{L, R}$ is the normal conductance of each junction, $F(\epsilon)=\int \mathrm{d} \omega(\partial f(\omega) / \partial \omega)[f(\omega+\epsilon-V)+f(\omega+\epsilon+V)]$ is a thermal smearing kernel arising from the Fermi distribution $f(\omega)$, whereas $N\left(\omega_{\alpha}\right)$ is the Bose distribution function. The planar geometry leads to the factor $\mathrm{E} 1(2 d / \xi)$, where E1 denotes the exponential integral function. This leads to an exponential decay of the non-local conductance when increasing $d$.

To understand the behaviour of $G_{L R}$ as a function of voltage it is convenient to first analyse the contribution arising from a given wavevector $q$. This is shown in Fig. 3a for temperature $T=10^{-2} \Delta$ and $\hbar \omega_{0}=0.4 \Delta$. The behaviour for the different wavectors is qualitatively similar: for $e V<\hbar \omega_{1}, \hbar \omega_{2}$ the EC processes dominate, whereas CAR becomes more important in the voltage window $\hbar \omega_{1}<e V<\hbar \omega_{2}$ and finally both contributions cancel for $e V>\hbar \omega_{2}$.

The sum of all contributions yields a non-local conductance that is dominated by EC processes at $V \rightarrow 0$ and decreases almost linearly with $V$ until $e V \simeq \hbar \omega_{0}$. Close to this point there is a change of sign in $G_{L R}$ indicating the dominance of CAR processes. We can therefore associate $\hbar \omega_{0}$ with the crossing energy from EC- to CAR-dominated regimes. Figure $3 \mathrm{~b}$ shows the voltage dependence of $G_{L R}$ for different temperatures. As can be observed, the imbalance between EC and CAR processes driven by the electromagnetic modes is less pronounced for increasing temperature. The characteristic temperature for the suppression of the non-local conductance is set by $\hbar \omega_{0} / k_{\mathrm{B}}$. Note that the temperature dependence arises mainly from the smearing of the Fermi factors. The overall features of the curves in Fig. 3, including the weak temperature dependence of the crossing energy, are in qualitative agreement with the results of ref. 11. Moreover, the magnitude of the non-local conductance predicted by our model is in reasonable agreement with the experimental values. For instance, the ratio $G_{L R} / G_{L, R}^{s}$ between the non-local and the direct conductances in the superconducting state at zero voltage and zero temperature is $0.75 \mathrm{E} 1(2 d / \xi)\left(E_{\mathrm{c}} \& \omega_{0} / \hbar c_{s}^{2}\right)$, which yields values $\sim 10^{-3}$ that are close to the experimental ones for the parameters estimated above. A more quantitative description of the experimental results may require the inclusion of higher order terms in the barrier transparency, which is beyond the scope of this work. Note, however, that weak tunnelling conditions are ideal for the observation of true CAR processes that would be masked by non-equilibrium effects when increasing the barrier transmission, as discussed in ref. 19. It is also worth mentioning recent experiments by Beckmann et al. ${ }^{20}$ where a behaviour similar to the one predicted by our theory is observed when decreasing the barrier transparency.

In summary, we have shown that electron interactions mediated by electromagnetic excitations lead to an imbalance between EC and CAR processes. Electromagnetic modes can either suppress CAR or EC processes depending on their spatial symmetry. Taking into account that these low-energy excitations are strongly dependent on the geometrical characteristics of the multiterminal device, these findings open the possibility to control non-local transport processes through a superconductor by an appropriate design of the experimental set-up. For instance, one possibility would be to introduce an additional tunnel junction inside the superconducting film in the planar double barrier geometry. This normal/superconducting/superconducting/normal nanostructure would enable us to control the dispersion relation of the electromagnetic modes by varying the Josephson coupling between the superconducting layers by means of a magnetic field. Similar effects could be achieved by means of layered superconductors (either high $T_{c}$ or organic compounds) that are known to exhibit bulk collective excitations with frequencies below the superconducting gap ${ }^{21}$. Let us finally point out that the high sensitivity of non-local transport to the electromagnetic modes could be used as a tool to analyse these excitations in hybrid nanostructures. 
Received 18 0ctober 2006; accepted 13 April 2007; published 13 May 2007.

\section{References}

1. Recher, P., Sukhorukov, E. V. \& Loss, D. Andreev tunneling, Coulomb blockade, and resonant transport of nonlocal spin-entangled electrons. Phys. Rev. B 63, 165314 (2001).

2. Chtchelkatchev, N. M., Blatter, G., Lesovik, G. B. \& Martin, T. Bell inequalities and entanglement in solid-state devices. Phys. Rev. B 66, 161320 (2002).

3. Bena, C., Vishveshwara, S., Balents, L. \& Fisher, M. P. A. Quantum entanglement in carbon nanotubes. Phys. Rev. Lett. 89, 037901 (2002).

4. Samuelsson, P., Sukhorukov, E. V. \& Büttiker, M. Orbital entanglement and violation of Bell inequalities in mesoscopic conductors. Phys. Rev. Lett. 91, 157002 (2003).

5. Prada, E. \& Sols, F. Entangled electron current through finite size normal-superconductor tunneling structures. Eur. Phys. J. B 40, 379-396 (2004).

6. Byers, J. M. \& Flatté, M. E. Probing spatial correlations with nanoscale two-contact tunneling. Phys. Rev. Lett. 74, 306-309 (1995).

7. den Hartog, S. G. et al. Transport in multiterminal normal-superconductor devices: Reciprocity relations, negative and nonlocal resistances, and reentrance of the proximity effect. Phys. Rev. Lett. 77, 4954-4957 (1996).

8. Deutscher, G. \& Feinberg, D. Coupling superconducting-ferromagnetic point contacts by Andreev reflections. Appl. Phys. Lett. 76, 487-489 (2000).

9. Mooij, J. E. \& Schön, G. Propagating plasma mode in thin superconducting filaments. Phys. Rev. Lett. 55, 114-117 (1985).

10. Beckmann, D. Weber, H. B. \& Löhneysen, H. v. Evidence for crossed Andreev reflection in superconductor-ferromagnet hybrid structures. Phys. Rev. Lett. 93, 197003 (2004).

11. Russo, S., Kroug, M., Klapwijk, T. M. \& Morpurgo, A. F. Experimental observation of bias-dependen nonlocal Andreev reflection. Phys. Rev. Lett. 95, 027002 (2005)

12. Falci, G., Feinberg, D. \& Hekking, F. W. J. Correlated tunneling into a superconductor in a multiprobe hybrid structure. Europhys. Lett. 54, 255-261 (2001).

13. Feinberg, D. Andreev scattering and cotunneling between two superconductor-normal metal interfaces: the dirty limit. Eur. Phys. J. B 36, 419-422 (2003).
14. Mélin, R. \& Feinberg, D. Sign of the crossed conductances at a ferromagnet/superconductor/ ferromagnet double interface. Phys. Rev. B 70, 174509-174518 (2004).

15. Hermele, M., Rafael, G., Fisher, M. P. A. \& Goldbart, P. M. Fate of the Josephson effect in thin-film superconductors. Nature Phys. 1, 117-121 (2005).

16. Orlando, T. P. \& Delin, K. E. Foundations of Applied Superconductivity (Addison-Wesley, Reading, 1991).

17. Gubin, A. I. et al. Dependence of magnetic penetration depth on the thickness of superconducting $\mathrm{Nb}$ thin films. Phys. Rev. B 72, 064503 (2005).

18. Parage, F., Doria, M. M. \& Buisson, O. Plasma modes in periodic two-dimensional superconducting-wire networks. Phys. Rev. B 58, R8921-R8924 (1998).

19. Cadden-Zimansky, P. \& Chandrasekhar, V. Non-local correlations in normal metal-superconducting systems. Phys. Rev. Lett. 97, 237003 (2006).

20. Beckmann, D. \& Lohneysen, H. v. Negative four-terminal resistance as a probe of crossed Andreev reflection. Preprint at <http://arxiv.org/abs/cond-mat/0609766> (2006).

21. Fertig, H. A. \& Das Sarma, S. Collective modes in layered superconductors. Phys. Rev. Lett. 65 1482-1485 (1990).

Acknowledgements

The authors acknowledge fruitful discussions and correspondence with D. Beckmann, A. Morpurgo, S. Russo, C. Urbina, D. Esteve, W. Herrera, R. C. Monreal, J. C. Cuevas and A. F. Volkov. Financial support by the Spanish M.E.C. under contract FIS2005-06255 is acknowledged. F.S.B. acknowledges funding by the Ramón y Cajal program.

Correspondence and requests for materials should be addressed to A.L.Y.

Supplementary Information accompanies this paper on www.nature.com/naturephysics.

Competing financial interests

The authors declare no competing financial interests.

Reprints and permission information is available online at http://npg.nature.com/reprintsandpermissions/ 